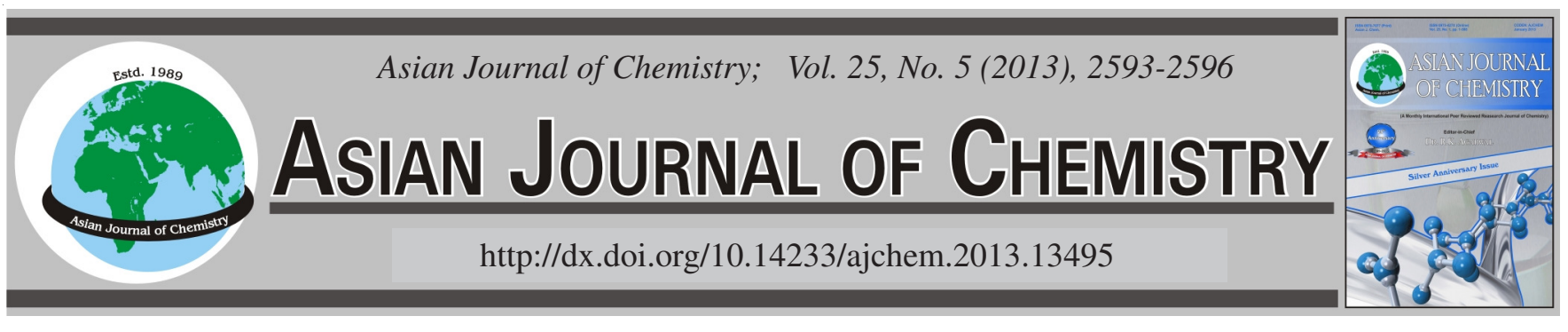

\title{
Synthesis and Antioxidant and Antibacterial Activities of Metal-Based Schiff Bases of Nicotinoyl, Isonicotinoyl and Benzoyl Hydrazides
}

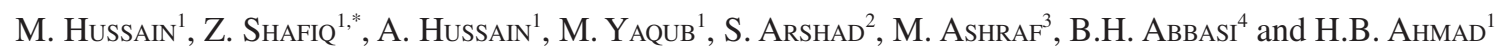

${ }^{1}$ Department of Chemistry, Bahauddin Zakariya University, Multan, Pakistan

${ }^{2}$ College of Conventional Medicine, The Islamia University of Bahawalpur, Bahawalpur, Pakistan

${ }^{3}$ Department of Biochemistry and Biotechnology, The Islamia University of Bahawalpur, Bahawalpur, Pakistan

${ }^{4}$ Department of Biotechnology, Quaid-e-Azam University, Islamabad, Pakistan

*Corresponding author: Fax: +92 61 9210138; Tel: +92 61 9210085; E-mail: z_shafiq@yahoo.com

\begin{abstract}
A series of 15 metal based [Cu(II), $\mathrm{Co}(\mathrm{II})$ and $\mathrm{Ni}(\mathrm{II})]$ complexes have been synthesized with novel N'-[-(5-hydroxy-2-nitrophenyl)methylidene]pyridine-4-carbohydrazide $\left(\mathrm{L}^{1}\right), \mathrm{N}^{\prime}-\left[-1-\left(2,5\right.\right.$-dihydroxyphenyl)ethylidene]pyridine-3-carbohydrazide $\left(\mathrm{L}^{2}\right), \mathrm{N}^{\prime}-[-1-(5$-chloro2-hydroxphenyl)ethylidene]pyridine-3-carbohydrazide $\left(\mathrm{L}^{3}\right), \mathrm{N}^{\prime}-\left[-1-(5\right.$-chloro-2-hydroxyphenyl)ethylidene $]$ pyridine-4-carbohydrazide $\left(\mathrm{L}^{4}\right)$ and N'-[-1-(2, 5-dihydroxyphenyl)ethylidene]-3-hydroxybenzohydrazide $\left(\mathrm{L}^{5}\right)$ Schiff bases and screened for antioxidant and cytotoxic activities. All these complexes exhibited strong antioxidant activity against DPPH radical. Antibacterial activity assay exhibited MIC $_{50}$ values of these compounds comparable to standard drugs for both Gram-positive and Gram-negative bacteria.
\end{abstract}

Key Words: Antibacterial activity, Antioxidant, Benzoyl hydrazides, Nicotinoyl hydrazides, Isonicotinoyl hydrazides, Schiff Bases.

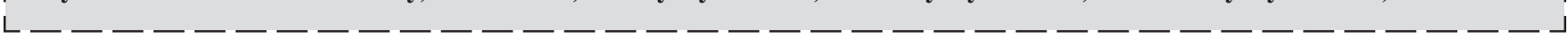

\section{INTRODUCTION}

Hydrazides have received a considerable attention due to their biological activity as tuberculostatic ${ }^{1}$, antibacterial ${ }^{2}$ and anticancer agents ${ }^{3}$. They have also been used in analytical chemistry as chelating agents ${ }^{4}$. Recently, a series of novel diflunisal hydrazide-hydrazone derivatives have demonstrated significant antimicrobial ${ }^{5}$ activity. Hydrazide-hydrazones exhibited antibacterial ${ }^{6}$, anticonvulsant ${ }^{7}$ and antitubercular ${ }^{8}$ activities. Very recently, we have reported the synthesis, antioxidant and cytotoxic activities ${ }^{9}$ of some novel hydrazidehydrazone Schiff bases and their [Cu(II), Co(II), Ni(II), Mn(II) and $\mathrm{Zn}(\mathrm{II})]$ complexes.

In the present studies, several new compounds have been synthesized to evaluate the effects of carbonyl compounds on the bioactivity of isoniazid, nicotinic acid hydrazide and 3-hydroxy benzoic acid hydrazide and the effect of metals like $\mathrm{Ni}(\mathrm{II}), \mathrm{Co}(\mathrm{II})$ and $\mathrm{Cu}(\mathrm{II})$ on the antioxidant activity of these compounds. The synthesis, antioxidant and antibacterial activity of hydrazide derivatives is therefore reported.

\section{EXPERIMENTAL}

Solvents used were of analytical grade and all metals (II) were used as chloride salts. IR spectra were recorded on 8400FTIR spectrophotometer using $\mathrm{KBr}$ disc and NMR spectra on a Bruker-300 MHz NMR spectrophotometer. UV-visible spectra were obtained in DMSO on a Hitachi U-2000 spectrophotometer. Conductance of the metal complexes was determined in DMSO on a Hitachi (Japan) YSI-32 model conductometer. Melting points were recorded on a Fischer Johns melting point apparatus and are uncorrected. The complexes were analyzed for their metal contents by EDTA titration.

Preparation of $\mathbf{L}^{\mathbf{1}}$ : To a hot stirred solution of isoniazid $(1.37 \mathrm{~g}, 0.01 \mathrm{~mol})$ in ethanol $(15 \mathrm{~mL}), 2$-nitro-5-hydroxybenzaldehyde $(1.67 \mathrm{~g}, 0.01 \mathrm{~mol})$ was added. The resultant mixture was heated under reflux. The reaction was monitored through TLC. After one hour reflux precipitates were obtained. The reaction mixture was further heated for $c a$. $0.5 \mathrm{~h}$. After completion of reaction, the mixture was cooled at room temperature. The solid was collected by suction filtration. The pink coloured precipitates were washed with hot ethanol, filtered and dried.

Preparation of $\mathbf{L}^{2}$ : To a hot stirred solution of 2,5dihydroxyacetophenone $(1.52 \mathrm{~g}, 0.01 \mathrm{~mol})$ in ethanol $(25 \mathrm{~mL})$ nicotinic acid hydrazide $(1.37 \mathrm{~g}, 0.01 \mathrm{~mol})$, was added. The resultant mixture was then heated under reflux. The reaction was monitored through TLC. After $0.5 \mathrm{~h}$, a solid product began to form. The reaction mixture was refluxed about 7-8 h. After the completion of reaction, the mixture was cooled at room temperature. The solid was collected by suction filtration. 
The yellow coloured precipitates were washed with hot ethanol, filtered and dried.

Preparation of $\mathbf{L}^{3}$ : To a hot stirred solution of 5-chloro2-hydroxyacetophenone (1.71 g, $0.01 \mathrm{~mol})$ in ethanol (25 mL), nicotinic acid hydrazide $(1.37 \mathrm{~g}, 0.01 \mathrm{~mol})$ was added. The resultant mixture was heated under reflux. The reaction was monitored through TLC. After half an hour, a solid product began to form. The reaction mixture was refluxed for 7-8 h. After completion of the reaction, the mixture was cooled at room temperature. The solid was collected by suction filtration. The light yellow coloured precipitates were washed with hot ethanol, filtered and dried.

Preparation of $\mathbf{L}^{4}$ : To a hot stirred solution of 5-chloro2-hydroxyacetophenone ( $1.71 \mathrm{~g}, 0.01 \mathrm{~mol})$ in ethanol (25 mL), isoniazid $(1.37 \mathrm{~g}, 0.01 \mathrm{~mol})$ was added. The resultant mixture was heated under reflux. The reaction was monitored through TLC. After half an hour a solid product began to form. The reaction mixture was refluxed for 7-8 h. After the completion of reaction, the mixture was cooled at room temperature. The solid was collected by suction filtration. The off white coloured precipitates were washed with hot ethanol, filtered and dried.

Preparation of $\mathbf{L}^{\mathbf{5}}$ : To a hot stirred solution of 2,5dihydroxyacetophenone $(1.52 \mathrm{~g}, 0.01 \mathrm{~mol})$ in ethanol $(25 \mathrm{~mL})$, 3 -hydroxybenzoichydrazide $(1.52 \mathrm{~g}, 0.01 \mathrm{~mol})$ was added. The resultant mixture was then heated under reflux. The reaction was monitored through TLC. After half an hour a solid product began to form. The reaction mixture was refluxed for 7-8 $\mathrm{h}$. After completion of the reaction, the mixture was cooled at room temperature. The solid was collected by suction filtration. The dark yellow coloured precipitates were washed with hot ethanol, filtered and dried.

N'-[-(5-Hydroxy-2-nitrophenyl)methylidene]pyridine4-carbohydrazide ( $\left.\mathbf{L}^{1}\right)$ : Yield $80 \%$, m.p. $290-292^{\circ} \mathrm{C}$; IR (KBr, $\left.v_{\max }, \mathrm{cm}^{-1}\right): 3361(\mathrm{NH}), 1660(>\mathrm{C}=\mathrm{O}), 1590(-\mathrm{HC}=\mathrm{N}-), 1340$, $1300\left(-\mathrm{NO}_{2}\right)$; ${ }^{1} \mathrm{H}$ NMR (DMSO) $\delta: 6.99(\mathrm{dd}, J=2.7 \& 9.0 \mathrm{~Hz}$, $\left.1 \mathrm{H}, \mathrm{C}_{12}-\mathrm{H}\right), 7.49\left(\mathrm{~d}, J=2.7 \mathrm{~Hz}, 1 \mathrm{H}, \mathrm{C}_{14}-\mathrm{H}\right), 7.86(\mathrm{~d}, J=5.7$ $\left.\mathrm{Hz}, 2 \mathrm{H}, \mathrm{C}_{3}-\mathrm{H}, \mathrm{C}_{5}-\mathrm{H}\right), 8.09$ (d, $\left.J=9.0 \mathrm{~Hz}, 1 \mathrm{H}, \mathrm{C}_{11}-\mathrm{H}\right), 8.80$ (d, $\left.J=5.7 \mathrm{~Hz}, 2 \mathrm{H}, \mathrm{C}_{2}-\mathrm{H}, \mathrm{C}_{6}-\mathrm{H}\right), 8.99$ (s, $\left.1 \mathrm{H}, \mathrm{N}=\mathrm{C}-\mathrm{H}\right), 11.16$ (s, $1 \mathrm{H}, \mathrm{NH}-\mathrm{CO}), 12.44\left(\mathrm{br}, \mathrm{C}_{13}-\mathrm{OH}\right) \mathrm{ppm}$; EIMS $(\mathrm{m} / \mathrm{z})=286\left(\mathrm{M}^{+}\right)$, 269, 240, 121, 106, 51.

N'-[1-(2,5-Dihydroxyphenyl)ethylidene]pyridine-3carbohydrazide $\left(\mathbf{L}^{2}\right)$ : Yield $78 \%$, m.p. $204-206^{\circ} \mathrm{C}$; IR ( $\mathrm{KBr}$, $\left.v_{\max }, \mathrm{cm}^{-1}\right): 3365(\mathrm{OH}), 3310(\mathrm{NH}), 1652(>\mathrm{C}=\mathrm{O}), 1590$, $(-\mathrm{N}=\mathrm{C}<),{ }^{1} \mathrm{H}$ NMR (DMSO) $\delta: 2.49\left(\mathrm{~s}, 3 \mathrm{H}, \mathrm{CH}_{3}-\mathrm{C}=\mathrm{N}\right), 6.73$ $\left(\mathrm{m}, 2 \mathrm{H}, \mathrm{C}_{12}-\mathrm{H}, \mathrm{C}_{13}-\mathrm{H}\right), 6.99$ (d, $\left.J=1.8 \mathrm{~Hz}, 1 \mathrm{H}, \mathrm{C}_{15}-\mathrm{H}\right), 7.62$ $\left(\mathrm{dd}, J=7.8 \& 5.1 \mathrm{~Hz}, 1 \mathrm{H}, \mathrm{C}_{5}-\mathrm{H}\right), 8.32(\mathrm{~d}, J=7.8 \mathrm{~Hz}, 1 \mathrm{H}$, $\left.\mathrm{C}_{4}-\mathrm{H}\right), 8.80\left(\mathrm{~d}, J=5.1 \mathrm{~Hz}, 1 \mathrm{H}, \mathrm{C}_{6}-\mathrm{H}\right), 8.97$ (br, $\left.\mathrm{C}_{14}-\mathrm{OH}\right), 9.09$ (s, 1H, $\left.\mathrm{C}_{2}-\mathrm{H}\right), 11.52$ (s, 1H, NH-CO), 12.48 (br, $\left.\mathrm{C}_{11}-\mathrm{OH}\right) \mathrm{ppm}$; $\operatorname{EIMS}(\mathrm{m} / \mathrm{z})=271\left(\mathrm{M}^{+}\right), 254,150,106,78,51$.

N'-[1-(5-Chloro-2-hydroxphenyl)ethylidene]pyridine3-carbohydrazide $\left(\mathbf{L}^{3}\right)$ : Yield $82 \%$, m.p. $160-162^{\circ} \mathrm{C}$; IR $(\mathrm{KBr}$, $\left.v_{\max }, \mathrm{cm}^{-1}\right): 3361(\mathrm{OH}), 3220$ (-NH-), 1648 (>C=O), 1600, (-C=N-); 1H NMR. (DMSO) fÔ: 2.51 (s, 3H, CH3-C=N), $6.96\left(\mathrm{~d}, J=8.7 \mathrm{~Hz}, 1 \mathrm{H}, \mathrm{C}_{12}-\mathrm{H}\right), 7.35(\mathrm{dd}, J=2.4 \& 8.7 \mathrm{~Hz}$, $\left.1 \mathrm{H}, \mathrm{C}_{13}-\mathrm{H}\right), 7.59\left(\mathrm{dd}, J=4.8 \& 7.8 \mathrm{~Hz}, 1 \mathrm{H}, \mathrm{C}_{5}-\mathrm{H}\right), 7.67(\mathrm{~d}$, $\left.J=2.4 \mathrm{~Hz}, 1 \mathrm{H}, \mathrm{C}_{15}-\mathrm{H}\right), 8.29$ (d, $\left.J=7.8 \mathrm{~Hz}, 1 \mathrm{H}, \mathrm{C}_{4}-\mathrm{H}\right), 8.79$ (d, $\left.J=4.8 \mathrm{~Hz}, 1 \mathrm{H}, \mathrm{C}_{6}-\mathrm{H}\right), 9.09$ (s, $\left.1 \mathrm{H}, \mathrm{C}_{2}-\mathrm{H}\right), 11.65(\mathrm{~s}, 1 \mathrm{H}$, NH-CO), 13.35 (br, 1H, C11-OH) ppm; EIMS (m/z) = 289 $\left(\mathrm{M}^{+}\right), 272,121,106,78,51$.
N'-[1-(5-Chloro-2-hydroxyphenyl)ethylidene]pyridine-4-carbohydrazide $\left(\mathbf{L}^{4}\right)$ : Yield $76 \%$, m.p. $216-218{ }^{\circ} \mathrm{C}$; IR (KBr, $\left.v_{\max }, \mathrm{cm}^{-1}\right): 3348(\mathrm{OH}), 3205(-\mathrm{NH}-), 1670(>\mathrm{C}=\mathrm{O})$, 1605, $(\mathrm{C}=\mathrm{N}),{ }^{1} \mathrm{H}$ NMR (DMSO) $\delta: 3.38\left(\mathrm{~s}, 3 \mathrm{H}, \mathrm{CH}_{3}-\mathrm{C}=\mathrm{N}\right)$, $6.96\left(\mathrm{~d}, J=9.0 \mathrm{~Hz}, 1 \mathrm{H}, \mathrm{C}_{12}-\mathrm{H}\right), 7.36(\mathrm{dd}, J=2.4 \& 9.0 \mathrm{~Hz}$, $\left.1 \mathrm{H}, \mathrm{C}_{13}-\mathrm{H}\right), 7.67\left(\mathrm{~d}, J=2.4 \mathrm{~Hz}, 1 \mathrm{H}, \mathrm{C}_{15}-\mathrm{H}\right), 7.85(\mathrm{~d}, J=5.7$ $\left.\mathrm{Hz}, 2 \mathrm{H}, \mathrm{C}_{3}-\mathrm{H}, \mathrm{C}_{5}-\mathrm{H}\right), 8.80$ (d, J = 5.7 Hz, 2H, C $\left.2-\mathrm{H}, \mathrm{C}_{6}-\mathrm{H}\right)$, 11.70 (s, 1H, NH-CO), 13.29 (br, 1H, C $\left.{ }_{11}-\mathrm{OH}\right)$ ppm; EIMS $(\mathrm{m} / \mathrm{z})=289\left(\mathrm{M}^{+}\right), 274,167,125,106,78,51$.

N'-[1-(2,5-Dihydroxyphenyl)ethylidene]-3-hydroxybenzohydrazide $\left(\mathbf{L}^{5}\right)$ : Yield $79 \%$, m.p. $204-206^{\circ} \mathrm{C}$; IR $(\mathrm{KBr}$, $\left.v_{\max }, \mathrm{cm}^{-1}\right): 3358(\mathrm{OH}), 3207$ (-NH-), $1650(>\mathrm{C}=\mathrm{O}), 1590$, $(>\mathrm{C}=\mathrm{N}-) ;{ }^{1} \mathrm{H}$ NMR (DMSO) $\delta: 3.39\left(\mathrm{~s}, 3 \mathrm{H}, \mathrm{CH}_{3}-\mathrm{C}=\mathrm{N}\right), 6.70$ (m, 2H, $\left.\mathrm{C}_{12}-\mathrm{H}, \mathrm{C}_{13}-\mathrm{H}\right), 6.98\left(\mathrm{~m}, 2 \mathrm{H}, \mathrm{C}_{6}-\mathrm{H}, \mathrm{C}_{15}-\mathrm{H}\right), 7.30(\mathrm{~m}$, $\left.3 \mathrm{H}, \mathrm{C}_{2}-\mathrm{H}, \mathrm{C}_{3}-\mathrm{H}, \mathrm{C}_{4}-\mathrm{H}\right), 8.95\left(\mathrm{~s}, 1 \mathrm{H}, \mathrm{C}_{14}-\mathrm{OH}\right), 9.83$ (s, $1 \mathrm{H}$, $\left.\mathrm{C}_{5}-\mathrm{OH}\right), 11.23$ (s, 1H, NH-CO), 12.61 (s, 1H, $\left.\mathrm{C}_{11}-\mathrm{OH}\right) \mathrm{ppm}$; EIMS $(\mathrm{m} / \mathrm{z})=286\left(\mathrm{M}^{+}\right), 271,148,121,160,93,65$.

\section{Preparation of metal (II) complexes}

Nickel(II) complex of $\mathbf{L}^{\mathbf{1}}$ : Nickel(II) chloride hexahydrate $(1 \mathrm{mmol}, 0.237 \mathrm{~g})$ in ethanol $(10 \mathrm{~mL})$ was added to a magnetically stirred solution of $\mathrm{L}^{1}$ ( $2 \mathrm{mmol}, 0.572 \mathrm{~g}$ ) in 1,4-dioxane (25 mL). The mixture was refluxed for $2 \mathrm{~h}$. Light yellow precipitates of the respective metal complex were formed after volume reduction by evaporation. The precipitates were filtered while hot, washed with ethanol and dried over silica gel in vacuum desiccators. All other complexes (2-15) were synthesized following the same method using the respective metal salts as chloride and ligands $\left(\mathrm{L}^{1}-\mathrm{L}^{5}\right)$.
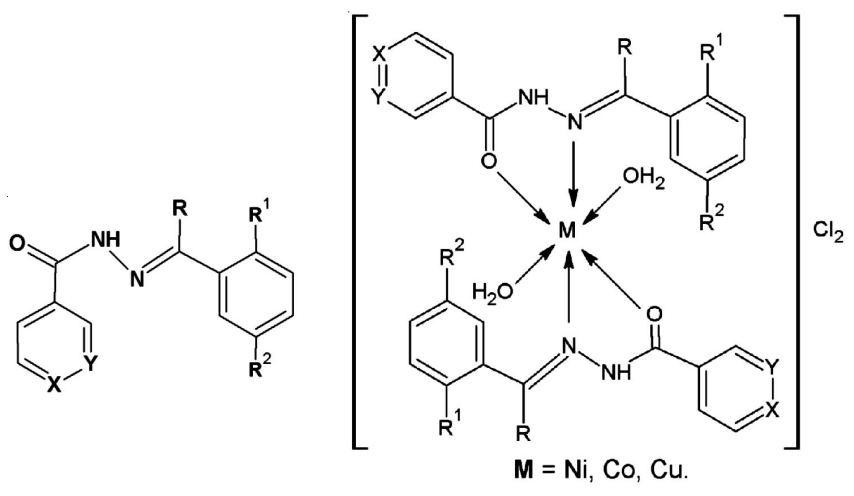

$$
\begin{aligned}
& \mathrm{L}^{1} \quad \mathrm{X}=\mathrm{N}, \quad \mathrm{Y}=\mathrm{CH}, \quad \mathrm{R}=\mathrm{H}, \quad \mathrm{R}^{1}=\mathrm{NO}_{2}, \quad \mathrm{R}_{2}=\mathrm{OH} \\
& \mathrm{L}^{2} \quad \mathrm{X}=\mathrm{CH}, \quad \mathrm{Y}=\mathrm{N}, \quad \mathrm{R}=\mathrm{CH}_{3}, \quad \mathrm{R}^{1}=\mathrm{OH}, \quad \mathrm{R}_{2}=\mathrm{OH} \\
& \mathrm{L}^{3} \quad \mathrm{X}=\mathrm{CH}, \quad \mathrm{Y}=\mathrm{N}, \quad \mathrm{R}=\mathrm{CH}_{3}, \quad \mathrm{R}^{1}=\mathrm{OH}, \quad \mathrm{R}_{2}=\mathrm{Cl} \\
& \mathrm{L}^{4} \quad \mathrm{X}=\mathrm{N}, \quad \mathrm{Y}=\mathrm{CH}, \quad \mathrm{R}=\mathrm{CH}_{3}, \quad \mathrm{R}^{1}=\mathrm{OH}, \quad \mathrm{R}_{2}=\mathrm{Cl} \\
& \mathrm{L}^{5} \quad \mathrm{X}=\mathrm{CH}, \quad \mathrm{Y}=\mathrm{C}-\mathrm{OH}, \quad \mathrm{R}=\mathrm{CH}_{3}, \quad \mathrm{R}^{1}=\mathrm{OH}, \quad \mathrm{R}_{2}=\mathrm{OH}
\end{aligned}
$$

Structure of transition metal(II) complexes

DPPH radical scavenging activity: The stable 1,1diphenyl-2-picrylhydrazyl radical (DPPH) was used for the determination of antioxidant activity as given ${ }^{10}$. Diffrent concentrations of compounds in DMSO (or a suitable solvent) were added at an equal volume $(5 \mu \mathrm{L})$ to $95 \mu \mathrm{L}$ of $100 \mu \mathrm{M}$ methanolic DPPH in a total volume of $100 \mu \mathrm{L}$ in 96 -well plates. The contents were mixed and incubated at $37^{\circ} \mathrm{C}$ for $0.5 \mathrm{~h}$. The absorbance was measured at $517 \mathrm{~nm}$. Propyl gallate and 3-t-butyl-4hydroxyanisole ( $t$-BHT) were used as standard antioxidants. The experiments were carried out in triplicates. $\mathrm{IC}_{50}$ value denotes the concentration of the sample which is required to 
scavenge $50 \%$ of DPPH free radicals. The activity was determined by the following formula and $\mathrm{IC}_{50}$ was were calculated using EZ-Fit 5 Perrella Scientific Inc. Amherst USA software, Per cent scavenging activity $=[100-($ Abs of test compound $/$ Abs of control) $\times 100]$

Antibacterial activity: Antibacterial activity was performed in sterile 96-wells microplates under aseptic environments ${ }^{11}$. The method is based on the principle that microbial cell number increases as the microbial growth proceeds in the log phase of growth which results in increased absorbance of broth medium. Four Gram-negative (Shigella sonnei, Escherichia coli, Pseudomonas aeruginosa and Salmonella typhi) and two Gram-positive bacteria (Bacillus subtilis, Staphylococcus aureus) were included in the study. The organisms were maintained on stock culture agar. The test samples with suitable solvent and dilution were pipetted into wells $(20 \mu \mathrm{g} / \mathrm{well})$. Overnight maintained fresh bacterial cultures after suitable dilution with fresh nutrient broth were poured into wells (180 $\mu \mathrm{L})$. The initial absorbance of the culture was strictly maintained between $0.12-0.19$ at $540 \mathrm{~nm}$. The total volume in each well was kept to $200 \mu \mathrm{L}$. The incubation was done at $37^{\circ} \mathrm{C}$ for $16-$ $24 \mathrm{~h}$ with lid on the microplate. The absorbance was measured at $540 \mathrm{~nm}$ using Synergy HT BioTekR USA microplate reader, before and after incubation and the difference was noted as an index of bacterial growth. The percent inhibition was calculated using the formula:

$$
\text { Inhibition }(\%)=100 \times(\mathrm{X}-\mathrm{Y}) / \mathrm{X}
$$

where $\mathrm{X}$ is absorbance in control with bacterial culture and $\mathrm{Y}$ is absorbance in test sample. Results are mean of triplicate ( $\mathrm{n}$ $=3$, mean \pm sem). Gentamycin and ampicilin were taken as standard. Minimum inhibitory concentration (MIC) was measured with suitable dilutions and results were calculated using EZFit 5 Perrella Scientific Inc. Amherst USA software and data expressed as $\mathrm{MIC}_{50}$.

\section{RESULTS AND DISCUSSION}

The Schiff bases $\mathrm{L}^{1}, \mathrm{~L}^{4}$ were prepared by refluxing equimolar $(0.01 \mathrm{~mol})$ quantities of isoniazid and the respective carbonyl compound, 5-hydroxy-2-nitrobenzaldehyde 5chloro-2-hydroxy acetophenone in ethanol. Schiff bases $\mathrm{L}^{2}$,
$\mathrm{L}^{3}$ were prepared by refluxing equimolar $(0.01 \mathrm{~mol})$ quantities of nicotinic acid hydrozide with 2,5-dihydroxy acetophenone and 5-chloro-2-hydroxy acetophenone, respectively in enthanol. This Schiff base $\mathrm{L}^{5}$ was prepared by refluxing equimolar $(0.01$ mol) quantity of 3-hydroxy benzoic hydrazide with 2,5dihydroxy acetophenone in ethanol. The precipitates formed were collected by suction filtration, washed with hot ethanol and dried. The purity of the products was checked on TLC plates and the spots were visualized under UV light at 250 and $360 \mathrm{~nm}$. The structures of all the synthesized bases were established through IR, Mass spectrum and ${ }^{1} \mathrm{H}$ NMR and micro analytical data.

The Schiff bases were further used for the complexation reaction with $\mathrm{Ni}$ (II), $\mathrm{Co}$ (II) and $\mathrm{Cu}$ (II) metal ions. All the metal complexes (1-15) of the Schiff bases were stable in air and decomposed above $190^{\circ} \mathrm{C}$ (Table-1). These were prepared by the stoichiometric reaction of the corresponding metal(II) chlorides with the Schiff base ligands in a molar ratio of 1:2. All the metal complexes are solid and intensely colored. They are insoluble in common organic solvents such as acetone, chloroform methanol and ethanol but soluble in DMSO and DMF. All the chelates exhibited high value of molar conductance (35.8-116 $\Omega^{-1} \mathrm{~cm}^{2} \mathrm{~mol}^{-1}$ ) determined in DMSO which showed their ionic and electrolyte nature.

IR spectra of ligands $\left(\mathrm{L}^{1}-\mathrm{L}^{5}\right)$ showed the absence of bands at $1725-1735 \mathrm{~cm}^{-1}$ due to carbonyl $(-\mathrm{C}=\mathrm{O})$ of aldehyde and ketones and at $3420-3440 \mathrm{~cm}^{-1}$ due to $-\mathrm{NH}_{2}$ stretching vibrations and instead a new band appeared at $1605-1590 \mathrm{~cm}^{-1}$ assigned ${ }^{12}$ to the azomethine $(\mathrm{C}=\mathrm{N})$ linkage. This suggested that amino and aldehyde moieties of the starting materials have been converted into their corresponding Schiff bases. The $(\mathrm{C}=\mathrm{O})$ of amide frequency was present at $1648-1675 \mathrm{~cm}^{-1}$. A comparison of the IR spectra of Schiff bases to the starting material indicated that Schiff base linkage $(\mathrm{C}=\mathrm{N})$ have been formed.

A comparison ${ }^{13}$ of the IR spectra of the Schiff bases $\left(\mathrm{L}^{1}\right.$ $\left.\mathrm{L}^{5}\right)$ and their metal(II) complexes (1-15) indicated that the ligands are coordinated to the metal atoms mainly in two ways, thus the ligand acting in a bidentate manner (Table-1). The band appearing at $1605-1590 \mathrm{~cm}^{-1}$ due to azomethine group was shifted to a lower frequency $c a .5-20 \mathrm{~cm}^{-1}$ indicating the participation of the azomethine $\mathrm{N}$ in the complexation with

TABLE-1

PHYSICAL AND ANALYTICAL DATA OF THE METAL(II) COMPLEXES (1-15)

\begin{tabular}{|c|c|c|c|c|}
\hline Compd. No. & Metal complex/m.f. (m.w.) & $\begin{array}{l}\text { Decom. } \\
\text { temp. }\left({ }^{\circ} \mathrm{C}\right)\end{array}$ & $\lambda_{\max }(\mathrm{nm})$ & $\operatorname{IR}\left(\mathrm{cm}^{-1}\right)$ \\
\hline 1 & {$\left[\mathrm{Ni}\left(\mathrm{L}^{1}\right)_{2}\left(\mathrm{H}_{2} \mathrm{O}\right)_{2}\right] \mathrm{Cl}_{2} ; \mathrm{C}_{26} \mathrm{H}_{24} \mathrm{~N}_{8} \mathrm{O}_{10} \mathrm{NiCl}_{2}[737.70]$} & 215 & 578 & $1643(\mathrm{CO}), 1582(\mathrm{C}=\mathrm{N}), 525(\mathrm{M}-\mathrm{O}), 423(\mathrm{M}-\mathrm{N})$ \\
\hline 2 & {$\left[\mathrm{Co}\left(\mathrm{L}^{1}\right)_{2}\left(\mathrm{H}_{2} \mathrm{O}\right)_{2}\right] \mathrm{Cl}_{2} ; \mathrm{C}_{26} \mathrm{H}_{24} \mathrm{~N}_{8} \mathrm{O}_{10} \mathrm{CoCl}_{2}$ [737.93] } & 224 & 552 & $1647(\mathrm{CO}), 1579(\mathrm{C}=\mathrm{N}), 533(\mathrm{M}-\mathrm{O}), 440(\mathrm{M}-\mathrm{N})$ \\
\hline 3 & {$\left[\mathrm{Cu}\left(\mathrm{L}^{1}\right)_{2}\left(\mathrm{H}_{2} \mathrm{O}\right)_{2}\right] \mathrm{Cl}_{2} ; \mathrm{C}_{26} \mathrm{H}_{24} \mathrm{~N}_{8} \mathrm{O}_{10} \mathrm{CuCl}_{2}$ [742.546] } & 219 & 497 & $1641(\mathrm{CO}), 1581(\mathrm{C}=\mathrm{N}), 537(\mathrm{M}-\mathrm{O}), 432(\mathrm{M}-\mathrm{N})$ \\
\hline 4 & {$\left[\mathrm{Ni}\left(\mathrm{L}^{2}\right)_{2}\left(\mathrm{H}_{2} \mathrm{O}\right)_{2}\right] \mathrm{Cl}_{2} ; \mathrm{C}_{28} \mathrm{H}_{30} \mathrm{~N}_{6} \mathrm{O}_{8} \mathrm{NiCl}_{2}[707.70]$} & 234 & 493 & $1641(\mathrm{CO}), 1577(\mathrm{C}=\mathrm{N}), 540(\mathrm{M}-\mathrm{O}), 438(\mathrm{M}-\mathrm{N})$ \\
\hline 5 & {$\left[\mathrm{Co}\left(\mathrm{L}^{2}\right)_{2}\left(\mathrm{H}_{2} \mathrm{O}\right)_{2}\right] \mathrm{Cl}_{2} ; \mathrm{C}_{28} \mathrm{H}_{30} \mathrm{~N}_{6} \mathrm{O}_{8} \mathrm{CoCl}_{2}$ [707.933] } & 227 & 523 & $1643(\mathrm{CO}), 1583(\mathrm{C}=\mathrm{N}), 541(\mathrm{M}-\mathrm{O}), 432(\mathrm{M}-\mathrm{N})$ \\
\hline 6 & {$\left[\mathrm{Cu}\left(\mathrm{L}^{2}\right)_{2}\left(\mathrm{H}_{2} \mathrm{O}\right)_{2}\right] \mathrm{Cl}_{2} ; \mathrm{C}_{28} \mathrm{H}_{30} \mathrm{~N}_{6} \mathrm{O}_{8} \mathrm{CuCl}_{2}[712.546]$} & 224 & 489 & $1645(\mathrm{CO}), 1582(\mathrm{C}=\mathrm{N}), 533(\mathrm{M}-\mathrm{O}), 440(\mathrm{M}-\mathrm{N})$ \\
\hline 7 & {$\left[\mathrm{Ni}\left(\mathrm{L}^{3}\right)_{2}\left(\mathrm{H}_{2} \mathrm{O}\right)_{2}\right] \mathrm{Cl}_{2} ; \mathrm{C}_{28} \mathrm{H}_{28} \mathrm{~N}_{6} \mathrm{O}_{6} \mathrm{NiCl}_{4}[743.70]$} & 197 & 561 & $1637(\mathrm{CO}), 1592(\mathrm{C}=\mathrm{N}), 534(\mathrm{M}-\mathrm{O}), 442(\mathrm{M}-\mathrm{N})$ \\
\hline 8 & {$\left[\mathrm{Co}\left(\mathrm{L}^{3}\right)_{2}\left(\mathrm{H}_{2} \mathrm{O}\right)_{2}\right] \mathrm{Cl}_{2} ; \mathrm{C}_{28} \mathrm{H}_{28} \mathrm{~N}_{6} \mathrm{O}_{6} \mathrm{CoCl}_{4}$ [743.933] } & 188 & 677 & $1638(\mathrm{CO}), 1595(\mathrm{C}=\mathrm{N}), 540(\mathrm{M}-\mathrm{O}), 437(\mathrm{M}-\mathrm{N})$ \\
\hline 9 & {$\left[\mathrm{Cu}\left(\mathrm{L}^{3}\right)_{2}\left(\mathrm{H}_{2} \mathrm{O}\right)_{2}\right] \mathrm{Cl}_{2} ; \mathrm{C}_{28} \mathrm{H}_{28} \mathrm{~N}_{6} \mathrm{O}_{6} \mathrm{CuCl}_{4}$ [748.546] } & 192 & 530 & $1640(\mathrm{CO}), 1594(\mathrm{C}=\mathrm{N}), 537(\mathrm{M}-\mathrm{O}), 438(\mathrm{M}-\mathrm{N})$ \\
\hline 10 & {$\left[\mathrm{Ni}\left(\mathrm{L}^{4}\right)_{2}\left(\mathrm{H}_{2} \mathrm{O}\right)_{2}\right] \mathrm{Cl}_{2} ; \mathrm{C}_{28} \mathrm{H}_{28} \mathrm{~N}_{6} \mathrm{O}_{6} \mathrm{NiCl}_{4}[743.70]$} & 242 & 601 & $1655(\mathrm{CO}), 1600(\mathrm{C}=\mathrm{N}), 534(\mathrm{M}-\mathrm{O}), 440(\mathrm{M}-\mathrm{N})$ \\
\hline 11 & {$\left[\mathrm{Co}\left(\mathrm{L}^{4}\right)_{2}\left(\mathrm{H}_{2} \mathrm{O}\right)_{2}\right] \mathrm{Cl}_{2} ; \mathrm{C}_{28} \mathrm{H}_{28} \mathrm{~N}_{6} \mathrm{O}_{6} \mathrm{CoCl}_{4}$ [743.93] } & 238 & 457 & $1657(\mathrm{CO}), 1592(\mathrm{C}=\mathrm{N}), 527(\mathrm{M}-\mathrm{O}), 433(\mathrm{M}-\mathrm{N})$ \\
\hline 12 & {$\left[\mathrm{Cu}\left(\mathrm{L}^{4}\right)_{2}\left(\mathrm{H}_{2} \mathrm{O}\right)_{2}\right] \mathrm{Cl}_{2} ; \mathrm{C}_{28} \mathrm{H}_{28} \mathrm{~N}_{6} \mathrm{O}_{6} \mathrm{CuCl}_{4}$ [748.546] } & 227 & 483 & $1657(\mathrm{CO}), 1597(\mathrm{C}=\mathrm{N}), 530(\mathrm{M}-\mathrm{O}), 433(\mathrm{M}-\mathrm{N})$ \\
\hline 13 & {$\left[\mathrm{Ni}\left(\mathrm{L}^{5}\right)_{2}\left(\mathrm{H}_{2} \mathrm{O}\right)_{2}\right] \mathrm{Cl}_{2} ; \mathrm{C}_{30} \mathrm{H}_{32} \mathrm{~N}_{4} \mathrm{O}_{10} \mathrm{NiCl}_{2}$ [737.70] } & 234 & 652 & $1641(\mathrm{CO}), 1585(\mathrm{C}=\mathrm{N}), 541(\mathrm{M}-\mathrm{O}), 440(\mathrm{M}-\mathrm{N})$ \\
\hline 14 & {$\left[\mathrm{Co}\left(\mathrm{L}^{5}\right)_{2}\left(\mathrm{H}_{2} \mathrm{O}\right)_{2}\right] \mathrm{Cl}_{2} ; \mathrm{C}_{30} \mathrm{H}_{32} \mathrm{~N}_{4} \mathrm{O}_{10} \mathrm{CoCl}_{2}$ [737.933] } & 240 & 511 & $1638(\mathrm{CO}), 1585(\mathrm{C}=\mathrm{N}), 537(\mathrm{M}-\mathrm{O}), 441(\mathrm{M}-\mathrm{N})$ \\
\hline 15 & {$\left[\mathrm{Cu}\left(\mathrm{L}^{5}\right)_{2}\left(\mathrm{H}_{2} \mathrm{O}\right)_{2}\right] \mathrm{Cl}_{2} ; \mathrm{C}_{30} \mathrm{H}_{32} \mathrm{~N}_{4} \mathrm{O}_{10} \mathrm{CuCl}_{2}$ [742.546] } & 231 & 489 & $1638(\mathrm{CO}), 1583(\mathrm{C}=\mathrm{N}), 540(\mathrm{M}-\mathrm{O}), 437(\mathrm{M}-\mathrm{N})$ \\
\hline
\end{tabular}




\begin{tabular}{|c|c|c|c|c|c|c|}
\hline \multirow{3}{*}{ Compd. No. } & \multicolumn{5}{|c|}{$\begin{array}{l}\text { TABLE-3 } \\
\text { F METAL(II) COMPLEXES (MEAN } \pm \text { SEM, } n=3 \text { ) }\end{array}$} & \\
\hline & \multicolumn{6}{|c|}{ Antibacterial activity MIC50 $(\mu \mathrm{g} / \mathrm{mL})$} \\
\hline & S. aureus & B. subtilis & S. typhi & E. coli & S. sonnei & P. aureginosa \\
\hline 2 & $13.92 \pm 0.14$ & - & - & $12.32 \pm 0.11$ & $13.09 \pm 0.12$ & - \\
\hline 3 & $12.33 \pm 0.09$ & $13.43 \pm 0.15$ & $13.02 \pm 0.32$ & $11.66 \pm 0.02$ & $11.29 \pm 0.12$ & $11.76 \pm 0.22$ \\
\hline 6 & - & $14.76 \pm 0.09$ & - & - & - & - \\
\hline 7 & - & - & $13.66 \pm 0.11$ & - & - & $12.07 \pm 0.01$ \\
\hline 8 & $12.66 \pm 0.15$ & $12.38 \pm 0.22$ & $14.06 \pm 0.38$ & $12.21 \pm 0.08$ & $14.33 \pm 0.33$ & $13.32 \pm 0.16$ \\
\hline 9 & $12.68 \pm 0.11$ & - & $12.12 \pm 0.60$ & $11.78 \pm 0.24$ & - & $11.62 \pm 0.38$ \\
\hline 12 & $13.23 \pm 0.93$ & $12.26 \pm 0.31$ & $12.29 \pm 0.26$ & - & $11.57 \pm 0.21$ & $12.03 \pm 0.08$ \\
\hline 15 & - & $13.18 \pm 0.16$ & $12.86 \pm 0.02$ & $12.39 \pm 0.25$ & - & - \\
\hline Gentamycin & $9.42 \pm 0.12$ & $10.36 \pm 0.13$ & $11.21 \pm 0.31$ & $9.29 \pm 0.02$ & $9.31 \pm 0.18$ & $10.89 \pm 0.11$ \\
\hline Ampicilin & $10.69 \pm 0.06$ & $11.66 \pm 0.14$ & $10.85 \pm 0.16$ & $11.32 \pm 0.13$ & $11.98 \pm 0.13$ & $12.33 \pm 0.15$ \\
\hline
\end{tabular}

the metal ion. The band at $1648-1675 \mathrm{~cm}^{-1}$ assigned to the $(-\mathrm{C}=\mathrm{O})$ group is also shifted to lower frequency by $c a .5-20$ $\mathrm{cm}^{-1}$ which is indicative of the involvement of the carbonyl group of hydrazide moiety in the coordination. Further conclusive evidence of the coordination of these Schiff bases with the metal ions was shown by the appearance of week low frequency new bands at $455-433$ and $540-525 \mathrm{~cm}^{-1}$. These are assigned $^{14}$ to the metal nitrogen $(\mathrm{M}-\mathrm{N})$ and metal oxygen (M-O) vibrations, respectively and were observed in the spectra of the metal complexes and not in the spectra of the uncomplexed Schiff bases, thus confirming the participation of $\mathrm{O}$ and $\mathrm{N}$ atoms in the coordination. All of the data established the fact that a conjugate chelate formed by ligand enolization exists in these complexes.

The NMR spectra of ligands were determined in DMSO. The ${ }^{1} \mathrm{H}$ NMR spectral data are reported along with the possible assignments. All the protons were found to be in their expected regions ${ }^{15}$. The conclusions drawn from these studies lend further support to the mode of bonding discussed in their IR spectra. It was also observed that DMSO did not have any coordinating effect on the spectra of ligands or their metal complexes.

Antioxidant and antibacterial activities: Compounds $\mathbf{1 3}$ and $\mathbf{1 5}$ showed maximum antioxidant activity against the

\begin{tabular}{ccc}
\multicolumn{3}{c}{ TABLE-2 } \\
\multicolumn{3}{c}{$\begin{array}{c}\text { ANTIOXIDANT ACTIVITY OF } \\
\text { COMPOUNDS BY DPPH METHOD }\end{array}$} \\
\hline Comp. No. & $\begin{array}{c}\text { DPPH scavenging } \\
\text { activity }(\%) \text { at } 0.5 \mathrm{mM}\end{array}$ & $\mathrm{IC}_{50}(\mu \mathrm{m})$ \\
\hline $\mathbf{1}$ & $42.86 \pm 2.13$ & - \\
$\mathbf{2}$ & $23.84 \pm 1.25$ & - \\
$\mathbf{3}$ & $87.33 \pm 2.45$ & $98.77 \pm 2.21$ \\
$\mathbf{4}$ & $48.23 \pm 1.65$ & - \\
$\mathbf{5}$ & $18.67 \pm 1.57$ & - \\
$\mathbf{6}$ & $46.31 \pm 1.87$ & - \\
$\mathbf{7}$ & $58.27 \pm 1.12$ & $227.45 \pm 4.96$ \\
$\mathbf{8}$ & $70.37 \pm 1.42$ & $161.12 \pm 2.68$ \\
$\mathbf{9}$ & $79.71 \pm 1.65$ & $71.25 \pm 2.06$ \\
$\mathbf{1 0}$ & $37.37 \pm 1.11$ & - \\
$\mathbf{1 1}$ & $65.06 \pm 1.24$ & $151.91 \pm 3.12$ \\
$\mathbf{1 2}$ & $72.61 \pm 1.46$ & $70.33 \pm 1.13$ \\
$\mathbf{1 3}$ & $82.58 \pm 1.43$ & $22.17 \pm 0.95$ \\
$\mathbf{1 4}$ & $18.93 \pm 0.79$ & - \\
$\mathbf{1 5}$ & $89.33 \pm 1.98$ & $16.75 \pm 0.72$ \\
$3-t$-BHA & $91.89 \pm 1.96$ & $44.12 \pm 2.31$ \\
Propyl gallate & $93.18 \pm 1.17$ & $32.21 \pm 0.83$ \\
\hline
\end{tabular}

Data is given both as percent activity and as $\operatorname{IC}_{50}(\mu \mathrm{M})$ of active compounds. free radical DPPH with lowest $\mathrm{IC}_{50}$ values, even lower than the control (Table-2). These results suggest highest antioxidant activity of these compounds. Compounds 3, 9, 12 possess antioxidant activity slightly higer than that of standard 3-tbutyl-4-hydroxyanisole and propyl gallate (compare $\mathrm{IC}_{50}$ values with that of standard) and are also good as antioxidant. However, compounds 8, 11 had higher $\mathrm{IC}_{50}$ values than that of control and possess low antioxidant activities (ca. 65-70\% activity).

When the antibacterial activities of these compounds were carried out with two gram positive and four gram negative bacteria, only selected compounds exhibited antibacterial activity. Lowest the $\mathrm{MIC}_{50}$ value, highest is the antibacterial activity. Compounds $\mathbf{3 , 8}, \mathbf{9}, 12$ and $\mathbf{1 5}$ possessed $\mathrm{MIC}_{50}$ values close to the standard drugs gentamycin and ampicilin (Table3). These compounds were active against both gram positive and gram negative bacteria. These results suggest the potential of these molecules as antibacterial agents and screening against large clinical isolates is suggested for further work.

\section{REFERENCES}

1. G.D. Yadav, S.S. Joshi and P.S. Lathi, Enzyme Microb. Technol., 36, 217 (2005).

2. R. Malhotra, M.S. Malik, J.P. Singh and K.S. Dhindsa, J. Inorg. Biochem., 45, 269 (1992).

3. H. Zhang, J. Drewe, B. Tseng, S. Kasibhatla and S.X. Cai, Bioorg. Med. Chem., 12, 3649 (2004).

4. D. Fournand, A. Arnoud and P. Galzy, J. Mol. Catal. B, 4, 77 (1998).

5. S.G. Kucukguzel, A. Mazi, F. Sahin, S. Ozturk and J. Stables, Eur. J. Med. Chem., 38, 1005 (2003).

6. S.G. Kucukguzel, E.E. Oruc, S. Rollas, F. Sahin and A. Ozbek, Eur. J. Med. Chem., 37, 197 (2002).

7. S.N. Pandeya, H. Manjula and J.P. Stables, Pharmazie, 56, 121 (2001).

8. S.G. Kucukguzel, S. Rollas, I. Kucukguzel and M. Kiraz, Eur. J. Med. Chem., 34, 1093 (1999).

9. Z. Shafiq, M. Hussain, M. Yaqub, K. Shafiq, M. Ashraf and H.B. Ahmad, Asian J. Chem, 24, 4623 (2012).

10. T.A. Koleva-van-Beek, J.P.H. Linssen, A. de Groot, L.N. Evstatieva, Phytochem. Anal., 13, 8 (2002).

11. M. Kaspady, V.K. Narayanaswamy, M. Raju and G.K. Rao, Lett. Drug Design Discov., 6, 21 (2009).

12. K. Nakamoto, Infrared Spectra of Inorganic and Coordination Compounds, New York, Wiley Interscience, edn. 2 (1970).

13. R.K. Agarwal, J. Indian Chem. Soc., 65, 448 (1988).

14. L.J. Bellamy, The Infrared Spectra of Complex Molecules, New York: John Wiley (1971).

15. W.W. Simmons, The Saddler Handbook of Protons NMR Spectra, Philadelphia, Saddler Research Laboratories Inc. (1978). 